\title{
Determination of the coefficients of melting, surfacing and loss of electrode metal
}

\author{
Smirnova, Zh. V. ${ }^{1}$, Svadbina T.V. ${ }^{2}$, Shmeleva N.V. ${ }^{3}$, Kutepova L.I. ${ }^{4}$, Vaganova O.I. ${ }^{5}$ \\ ${ }^{1}$ Minin Nizhny Novgorod State Pedagogical University (Minin University), Nizhny Novgorod, Russian Federation \\ z.v.smirnova@mininuniver.ru \\ ${ }^{2}$ Minin Nizhny Novgorod State Pedagogical University (Minin University), Nizhny Novgorod, Russian Federation \\ nhl_@list.ru \\ ${ }^{3}$ Minin Nizhny Novgorod State Pedagogical University (Minin University), Nizhny Novgorod, Russian Federation \\ Shmeleva_nv@mininuniver.ru \\ ${ }^{4}$ Minin Nizhny Novgorod State Pedagogical University (Minin University), Nizhny Novgorod, Russian Federation \\ lubovkutepova@mail.ru \\ ${ }^{5}$ Minin Nizhny Novgorod State Pedagogical University (Minin University), Nizhny Novgorod, Russian Federation \\ vaganova_o@mail.ru
}

\begin{abstract}
The article describes the process of determining the coefficients of melting, surfacing and loss of electrode metal. Standard formulas for solving these problems are given. A schematic example of the steps of surfacing by drip and jet transfer of electrode metal is given.
\end{abstract}

Key words: electrode metal, melting coefficient, surfacing, metal.

\section{INTRODUCTION}

When repairing rolling stock, welding and surfacing are parts made mainly of structural low-carbon, medium- and low-alloy rolled and cast steels. Carbon and alloying elements that make up steel have a significant effect on weldability and divide the latter into four groups: good, satisfactory, limited and poor weldability. Therefore, when developing a technological process, first of all, it is necessary to evaluate the weldability of the metal of the product being restored and to assign (if necessary) certain operations that reduce or exclude the negative impact of certain components on the quality of the restored layer [1]. The productivity of the process of electric fusion welding per unit time, depending on the welding method, is estimated differently with different welding methods:

a) when welding with a consumable electrode, it can be estimated by the mass of molten metal (Mp) or by the mass of deposited metal (Mn), defined as the excess mass of the product after welding;

b) when welding with a non-consumable electrode - by weight of the additional deposited metal (MD) of the welding wire;

c) when welding with a non-consumable electrode without introducing an additional welding wire, by mass of molten metal (mp) [2].

\section{RESEARCH}

The mass of molten electrode metal during the burning of the arc is determined by the formula.

$$
M_{p}=\alpha_{p} \cdot I_{s v} \cdot t_{0}
$$

A. where $\alpha_{p}$ - electrode metal melting coefficient, showing the mass of electrode metal in grams, molten by a welding current of $1 \mathrm{~A}$ per unit time (1 hour)

B. $; \frac{\Gamma}{\mathrm{A} * h}$

$I_{s v}-$ welding current, A;

$t_{0}$ - main welding time (arc burning time), $\mathrm{h}$

Hence, the melting coefficient $(\alpha p)$ is determined from the expression:

$$
\alpha_{p}=\frac{M_{P}}{I c B \cdot t_{0}}\left(\frac{\Gamma}{A-\varphi}\right)
$$

The performance of the melting of the electrode metal for 1 hour of arc burning will be:

$$
\Pi p=\alpha_{p} \cdot \operatorname{Isv}(\Gamma / h)
$$

Since during welding a part of the electrode metal is lost by evaporation and spraying, the mass of the deposited metal will be less than the mass of the molten electrode metal [3].

The mass of the deposited metal $(\mathrm{Mn})$ can be determined by the formula:

$$
\mathrm{MH}=\alpha_{H} \cdot I_{s v} \cdot t_{0}
$$

Hence, the deposition coefficient ( $\alpha$ n) is determined from the expression:

$$
\alpha_{\mu}=\frac{\mathrm{M} h}{I c b \cdot t_{0}} \quad\left(\frac{\Gamma}{A \cdot y}\right)
$$

The deposition coefficient shows how much metal in grams from the consumable electrode under the influence of a welding current of $1 \mathrm{~A}$ will be transferred to the base metal per unit time (1 hour).

The deposition rate for 1 hour of arc burning is:

$$
\Pi_{H}=\alpha_{H} \cdot I_{z v} \quad(\Gamma / h)
$$

Since part of the electrode metal in the welding process is lost by evaporation and spraying, in order to correctly 
determine the number of required electrodes or electrode wire, the loss of electrode metal should be taken into account [4].

The loss of electrode metal is estimated by the loss coefficient $(\varphi)$, determined by the formula:

$$
\varphi=\frac{\alpha_{\rho}-\alpha_{H}}{\alpha_{\rho}} \cdot 100 \%
$$

either

$$
\alpha_{\mu}=\alpha_{\rho}(1-\varphi)
$$

From equation (8), it is possible to determine the deposition coefficient, knowing the melting and loss coefficients [5].

\section{RESULT AND DISCUSSION}

In the process of research, we carried out work to determine the deposition and loss of fumes and spatter during manual arc welding.

In the process of manual arc welding, the processes of melting of the electrode metal occur, its transfer and surfacing on the surface of the welded or deposited part. Metal transfer can occur either discretely in droplets or in a continuous stream, as shown in Figure 1. Drop transfer of metal is observed at a low current density, jet transfer at high welding currents and a small diameter of the electrode wire [6].
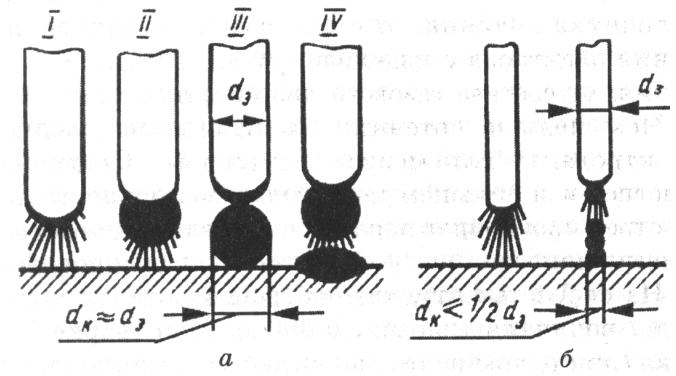

Figure 1: Stages of surfacing by drip (a) and jet transfer of electrode metal:

I - a burning arc begins to melt the metal; II — formation of a drop of molten metal; III - a drop flows down onto the metal, closing the arc gap; IV - restoration of the arc and the formation of the next drop; $\mathrm{dE}, \mathrm{dK}$ are the diameters of the drop electrode.

The main values characterizing the welding and surfacing process are:

- metal fusion coefficient $\alpha_{P}$,

- deposition rate $\alpha_{H}$,

- performance of fusion of electrodes $\Pi_{P}$,

- $\quad$ surfacing performance $\Pi_{H}$,

- loss ratio $\psi$.

The loss coefficient depends on the composition of the electrode and its coating, on the welding mode and the type of weld. For example, the loss coefficient increases with increasing current density and arc length. Usually value $\psi$ lies in the range from 1 to $3 \%$ when submerged arc welding; from 3 to $6 \%$ when welding in shielding gases; from 5 to $10 \%$ when welding with thick-coated electrodes; from 10 to $20 \%$ when welding with thinly coated electrodes.

At values greater than $20 \%$ of the loss coefficient, welding with electrodes is not practical.

Melting and surfacing coefficients are used to determine electrode consumption and normalize welding time.

If an electrode with an average coating thickness with a deposition coefficient of 8-9 g / Ah is used for welding, then welding with such electrodes can be produced in absolutely any spatial position.

Electrodes with a coating of large thickness are characterized by high performance - their deposition coefficient is up to $12 \mathrm{~g} / \mathrm{Ah}$. They can also, by and large, be used in any spatial position, but they will show the greatest efficiency when welding in the lower position, and the seam should have a sufficiently large length [7].

Electrodes with a coating of increased thickness belong to the class of high-performance - in them, the deposition coefficient reaches $18 \mathrm{~g} / \mathrm{Ah}$. [8]. This type of electrodes can be used exclusively for welding in the lower position, and the welds should be longer in this case - in this case, the use of the electrode will be characterized by maximum efficiency.

\section{CONCLUSION}

So, knowledge of the coefficient of electrode deposition is quite important during welding. The quality of preparation for welding works depends on the deposition coefficient - namely, the preparation of the required number of electrodes, which ensures the continuity of work, and the quality of the welded joint itself, since, based on knowledge of the deposition coefficient, the welder can determine in which position he is best to produce work and how long it will take.

\section{REFERENCES}

1. S. F. Andronov, B. M. Garipov Electrocontact surfacing of powder polymer materials // Welding production. - 2000. - No. 5. - from. 6 - 7.

2. V. M. Karpenko, V. T. Katrenko, candidates tech. sciences, V. A. Presnyakov, engineer. Electrocontact surfacing using powder materials enclosed in a metal shell // Automatic welding. - 1999. - No. 5. - from. 56 - 59.

3. V. M. Karpenko, V. T. Katrenko, V. A. Presnyakov Electrocontact surfacing of powder materials in a metal shell Kramatorsk .: KII, 1999, 126, p.3. Shuvalov V. M. "Geophysical research methods and interpretation of geophysical data" Perm - 2010.

4. Kutepova L.I., Parshakova A.M. Modern computer technology in the design of linear structures and engineering surveys //

5.Leonenko M.V. The methodology for assessing karst hazard according to geophysical methods (for example, the Nizhny Novgorod region). The dissertation for the degree of candidate of geological and mineralogical sciences. M., 1996.

6.Smirnova Zh.V., Bushueva V.V. Stages of quality indicators in construction // In the collection: Prospects for the development of science and education. Collection of scientific papers on the materials of the International 
Smirnova, Zh. V et al., International Journal of Emerging Trends in Engineering Research, 8(7), July 2020, $3824-3826$

scientific-practical conference: in 7 parts. LLC "ARConsult". 2013.S. 51-52

7. Santhosh , K. Hari Kishore , G. Pavani Lakshmi , G.Kushwanth4 , P. Rama Krishna Dharma Teja , R. S. Ernest Ravindran, Sree Vardhan Cheerala , M. Ravi Kumar Detection of Heavy Metal Ions using Star-Shaped Microfluidic Channel Chella. //International Journal of Emerging Trends in Engineering Research. Volume 7, No. 12 December 2019.

https://doi.org/10.30534/ijeter/2019/067122019

8. O. Amer, A. Salem, A Novel Leach Routing Protocol using Genetic Algorithm for Wireless Sensor Networks, International Journal of Advanced Trends in Computer Science and Engineering, Volume 8, No.5, September - October 2019

https://doi.org/10.30534/ijatcse/2019/37852019 